\title{
Correction to: Matrix versions of the Hellinger distance
}

\section{Rajendra Bhatia ${ }^{1}$. Stephane Gaubert ${ }^{2}$ - Tanvi Jain ${ }^{3}$}

Published online: 11 October 2019

(c) Springer Nature B.V. 2019

\section{Correction to: Letters in Mathematical Physics (2019) 109:1777-1804 https://doi.org/10.1007/s11005-019-01156-0}

Theorem 9 in our paper [1] is wrong. The statement should be replaced by the following:

Theorem When $d=d_{3}$, the minimum in (13) is attained at a unique point $X$ which is the solution of the matrix equation

$$
X^{2}=\frac{2}{\pi} \sum_{j=1}^{m} w_{j} \int_{0}^{\infty}\left(\lambda X^{-1}+A_{j}^{-1}\right)^{-2} \sqrt{\lambda} d \lambda .
$$

This minimiser is the $1 / 2$-power mean $Q_{1 / 2}$ given by (14) if $Q_{1 / 2}$ commutes with every $A_{j}$. In particular, the minimiser is $Q_{1 / 2}$ if

(i) All $A_{j}$ 's commute, or

(ii) $Q_{1 / 2}=I$.

An outline of Proof Let

$$
f(X)=\sum_{j=1}^{n} w_{j} \Phi_{3}\left(X, A_{j}\right)
$$

The original article can be found online at https://doi.org/10.1007/s11005-019-01156-0.

\footnotetext{
$凶$ Tanvi Jain

tanvi@isid.ac.in

Rajendra Bhatia

rajendra.bhatia@ashoka.edu.in

Stephane Gaubert

Stephane.Gaubert@inria.fr

1 Ashoka University, Sonepat, Haryana 131029, India

2 INRIA and CMAP, Ecole Polytechnique, CNRS, 91128 Palaiseau, France

3 Indian Statistical Institute, New Delhi 110016, India
} 
be the objective function (13) of [1]. Using Proposition 1 we see that

$$
\begin{aligned}
D f(X)(Y) & =\operatorname{tr}\left(Y-2 \sum_{j=1}^{m} w_{j} \int_{0}^{\infty}\left(\lambda+X A_{j}^{-1}\right)^{-1} Y\left(\lambda+A_{j}^{-1} X\right)^{-1} \mathrm{~d} \nu(\lambda)\right) \\
& =\operatorname{tr}\left(\left(I-2 \sum_{j=1}^{m} w_{j} \int_{0}^{\infty}\left(\left(\lambda+X A_{j}^{-1}\right)\left(\lambda+A_{j}^{-1} X\right)\right)^{-1} \mathrm{~d} \nu(\lambda)\right) Y\right) .
\end{aligned}
$$

Hence $X_{0}$ is a critical point of $f$ if and only if $X_{0}$ satisfies the matrix equation

$$
I=2 \sum_{j=1}^{m} w_{j} \int_{0}^{\infty}\left(\left(\lambda+X A_{j}^{-1}\right)\left(\lambda+A_{j}^{-1} X\right)\right)^{-1} \mathrm{~d} \nu(\lambda) .
$$

Taking congruence with $X$ on both sides, we see that this equation is equivalent to (1).

The function in (13) is strictly convex. So it has at most one critical point. This entails the uniqueness of the solution to (1). The existence of a solution follows from the Brouwer fixed point theorem. The argument is similar to that in our proof of Theorem 10.

On differentiating (25) of [1], we get

$$
\frac{1}{2} x^{-1 / 2}=\int_{0}^{\infty} \frac{1}{(\lambda+x)^{2}} \mathrm{~d} \nu(\lambda)
$$

So if $Q_{1 / 2} A_{j}^{-1}=A_{j}^{-1} Q_{1 / 2}$ for all $1 \leqslant j \leqslant m$, from (2) and (3) we get

$$
\begin{aligned}
I & =Q_{1 / 2}^{1 / 2} Q_{1 / 2}^{-1 / 2}=\sum_{j=1}^{m} w_{j}\left(A_{j}^{1 / 2} Q_{1 / 2}^{-1 / 2}\right) \\
& =\sum_{j=1}^{m} w_{j}\left(A_{j} Q_{1 / 2}^{-1}\right)^{1 / 2} \\
& =2 \sum_{j=1}^{m} w_{j} \int_{0}^{\infty}\left(\lambda+A_{j}^{-1} Q_{1 / 2}\right)^{-2} \mathrm{~d} v(\lambda) \\
& =2 \sum_{j=1}^{m} w_{j} \int_{0}^{\infty}\left(\left(\lambda+Q_{1 / 2} A_{j}^{-1}\right)\left(\lambda+A_{j}^{-1} Q_{1 / 2}\right)\right)^{-1} \mathrm{~d} v(\lambda) .
\end{aligned}
$$

This proves the second statement of the theorem.

We became aware of the mistake in Theorem 9 of [1] from the arxiv preprint [2]. The authors there give a correct version (in a different notation) and also give an example to show that the barycentre obtained in (1) does not reduce to formula (17) in our paper [1]. 


\section{References}

1. Bhatia, R., Gaubert, S., Jain, T.: Matrix versions of the Hellinger distance. Lett. Math. Phys. 109, 1777-1804 (2019)

2. Pitrik, J., Virosztek, D.: Quantum Hellinger distances revisited. arXiv:1903.10455v3

Publisher's Note Springer Nature remains neutral with regard to jurisdictional claims in published maps and institutional affiliations. 